\title{
Spatiotemporal characteristics and primary influencing factors of typical dengue fever epidemics in China
}

\author{
Lan Zheng ${ }^{1,2,3,4}$, Hong-Yan Ren ${ }^{2^{*}}$, Run-He Shi ${ }^{1,3,4^{*}}$ and Liang Lu ${ }^{5}$
}

\begin{abstract}
Background: Dengue fever (DF) is a common mosquito-borne viral infectious disease in the world, and increasingly severe DF epidemics in China have seriously affected people's health in recent years. Thus, investigating spatiotemporal patterns and potential influencing factors of DF epidemics in typical regions is critical to consolidate effective prevention and control measures for these regional epidemics.
\end{abstract}

Methods: A generalized additive model (GAM) was used to identify potential contributing factors that influence spatiotemporal epidemic patterns in typical DF epidemic regions of China (e.g., the Pearl River Delta [PRD] and the Border of Yunnan and Myanmar [BYM]). In terms of influencing factors, environmental factors including the normalized difference vegetation index (NDVI), temperature, precipitation, and humidity, in conjunction with socioeconomic factors, such as population density (Pop), road density, land-use, and gross domestic product, were employed.

Results: DF epidemics in the PRD and BYM exhibit prominent spatial variations at $4 \mathrm{~km}$ and $3 \mathrm{~km}$ grid scales, characterized by significant spatial clustering over the Guangzhou-Foshan, Dehong, and Xishuangbanna areas. The GAM that integrated the Pop-urban land ratio (ULR)-NDVI-humidity-temperature factors for the PRD and the ULR-Road density-NDVI-temperaturewater land ratio-precipitation factors for the BYM performed well in terms of overall accuracy, with Akaike Information Criterion values of 61859.89 and 826.65, explaining a total variance of 83.4 and 97.3\%, respectively. As indicated, socioeconomic factors have a stronger influence on DF epidemics than environmental factors in the study area. Among these factors, Pop (PRD) and ULR (BYM) were the socioeconomic factors explaining the largest variance in regional epidemics, whereas NDVI was the environmental factor explaining the largest variance in both regions. In addition, the common factors (ULR, NDVI, and temperature) in these two regions exhibited different effects on regional epidemics.

Conclusions: The spatiotemporal patterns of DF in the PRD and BYM are influenced by environmental and socioeconomic factors, the socioeconomic factors may play a significant role in DF epidemics in cases where environmental factors are suitable and differ only slightly throughout an area. Thus, prevention and control resources should be fully allocated by referring to the spatial patterns of primary influencing factors to better consolidate the prevention and control measures for DF epidemics.

Keywords: Spatiotemporal pattern, China, Dengue fever, Generalized additive model, Socioeconomic factor, Environmental factor

\footnotetext{
* Correspondence: renhy@|reis.ac.cn; renhy@igsnrr.ac.cn;

rhshi@geo.ecnu.edu.cn

${ }^{2}$ State Key Laboratory of Resources and Environmental Information System,

Institute of Geographic Sciences and Natural Resources Research, Chinese

Academy of Sciences, Beijing, China

${ }^{1}$ Key Laboratory of Geographic Information Science, Ministry of Education,

East China Normal University, Shanghai, China

Full list of author information is available at the end of the article
}

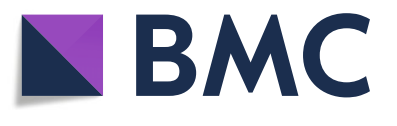

(c) The Author(s). 2019 Open Access This article is distributed under the terms of the Creative Commons Attribution 4.0 International License (http://creativecommons.org/licenses/by/4.0/), which permits unrestricted use, distribution, and

reproduction in any medium, provided you give appropriate credit to the original author(s) and the source, provide a link to the Creative Commons license, and indicate if changes were made. The Creative Commons Public Domain Dedication waiver (http://creativecommons.org/publicdomain/zero/1.0/) applies to the data made available in this article, unless otherwise stated. 


\section{Multilingual abstracts}

Please see Additional file 1 for translations of the abstract into the five official working languages of the United Nations.

\section{Background}

Dengue fever (DF) is an acute infectious disease caused by the dengue virus, which is transmitted by Aedes albopictus and Aedes aegypti [1]. Approximately one-third of the global population is exposed to DF, which is widely endemic in tropical and subtropical areas, especially in Southeast Asia, the Western Pacific, and southern Africa [2]. In recent years, the increasing incidence and range of DF epidemics have had a serious impact on people's health and lives, and DF has become a public health problem that should not be underestimated.

In the mainland of China, DF is currently a localized epidemic caused by imported cases. No case was reported in China from 1949 to 1977 until an outbreak occurred in Guangdong Province in 1978, since then, China's DF epidemic has been intermittent [1]. With the acceleration of globalization and China's increasingly frequent international exchanges, the prevalence of DF induced by imported cases has increased substantially [36]. In recent years, DF epidemics have frequently occurred not only in southern China [7-9] but also in some inland areas, such as Henan (Xuchang) and Shandong (Jining) [10]. Overall, China's DF epidemic has shown increasingly shorter time intervals and a wider spread. In southern China, some typical regions with frequent DF epidemics have developed [11-14], especially in the Pearl River Delta (PRD) and the Border of Yunnan and Myanmar (BYM), and the local DF cases in the PRD and BYM accounted for $97.06 \%$ of cases nationwide from 2010 to 2014.

In the absence of effective vaccines, domestic and foreign scholars have conducted a large number of studies on factors, that affect the spread and prevalence of DF epidemics, such as the dengue virus, mosquito vectors, susceptible population, and environmental and socioeconomic factors [15-18]. Among these factors, environmental conditions, such as climate, hydrology, and vegetation, mainly affect the activity of the dengue virus, the breeding environment, and mosquito vector activity [19-21]. Socioeconomic factors, such as population density, land use, transportation convenience, residents' income level, and living habits, play an important role in DF epidemics by changing both the probability of bites from the mosquito vectors and their activities [22-25]. Previous studies on the epidemic scale, spatiotemporal characteristics, and influencing factors of DF have deepened our understanding of DF characteristics in China $[1,19]$. However, additional knowledge is needed regarding the spatial-temporal characteristics of the epidemic in China's typical DF epidemic areas and the differences in influencing factors. Our study was performed to 1) analyse the spatial-temporal pattern of DF epidemics in two regions and 2) use a generalized additive model (GAM) to analyse and compare the main influencing factors affecting the spatial disparities of DF in the two regions. The results of this study will provide important support for strengthening the prevention and control of DF outbreaks in the PRD and BYM and raising the level of prevention of DF risk.

\section{Methods}

Study area

The PRD $\left(111^{\circ} 28^{\prime}-114^{\circ} 42^{\prime} \mathrm{E}, 22^{\circ} 16^{\prime}-23^{\circ} 57^{\prime} \mathrm{N}\right)$ in the Guangdong Province is located at the Pearl River estuary and includes nine cities, such as Guangzhou and Foshan (Fig. 1). This area represents one of the major hubs for China's economic growth and is one of the most urbanized regions in the world. In addition, the PRD has a high population of 58.74 million, and the gross domestic product (GDP) per capita was approximately RMB 107000 yuan in 2015. The PRD also has a subtropical monsoon ocean climate that is humid and warm and has abundant sunshine hours throughout the year [26].

The BYM $\left(97^{\circ} 56^{\prime}-101^{\circ} 34^{\prime} \mathrm{E}, 21^{\circ} 28^{\prime}-24^{\circ} 43^{\prime} \mathrm{N}\right)$ referred to in our study mainly consists of Xishuangbanna, Dehong, and parts of Lincang and Pu'er in Yunnan Province. It is located on the boundary of Myanmar and Laos and presents several important international trade ports. In addition, this region has a population of approximately 4.4 million and a 2015 GDP per capita of approximately RMB 25000. Limited differences in temperature are observed throughout the year, although a large temperature difference occurs between day and night. The wet and dry seasons are distinct, and the water system in the area is developed.

\section{Data collection \\ DF incidence data}

Records of observed DF cases from 2010 to 2014 were obtained from the China Notifiable Disease Surveillance System, and the data included age, gender, occupation, date of onset, and type of diagnosis. In this study, only local cases were used to analyse the spatial-temporal characteristics of the local epidemic and the relationship between the pattern of the epidemic and local variables (environmental and socioeconomic). The DF cases were spatially located with geocoding (http://www.gpsspg.com/xGeocoding/) to enable calculation of the number of DF cases on different spatial grid scales. 


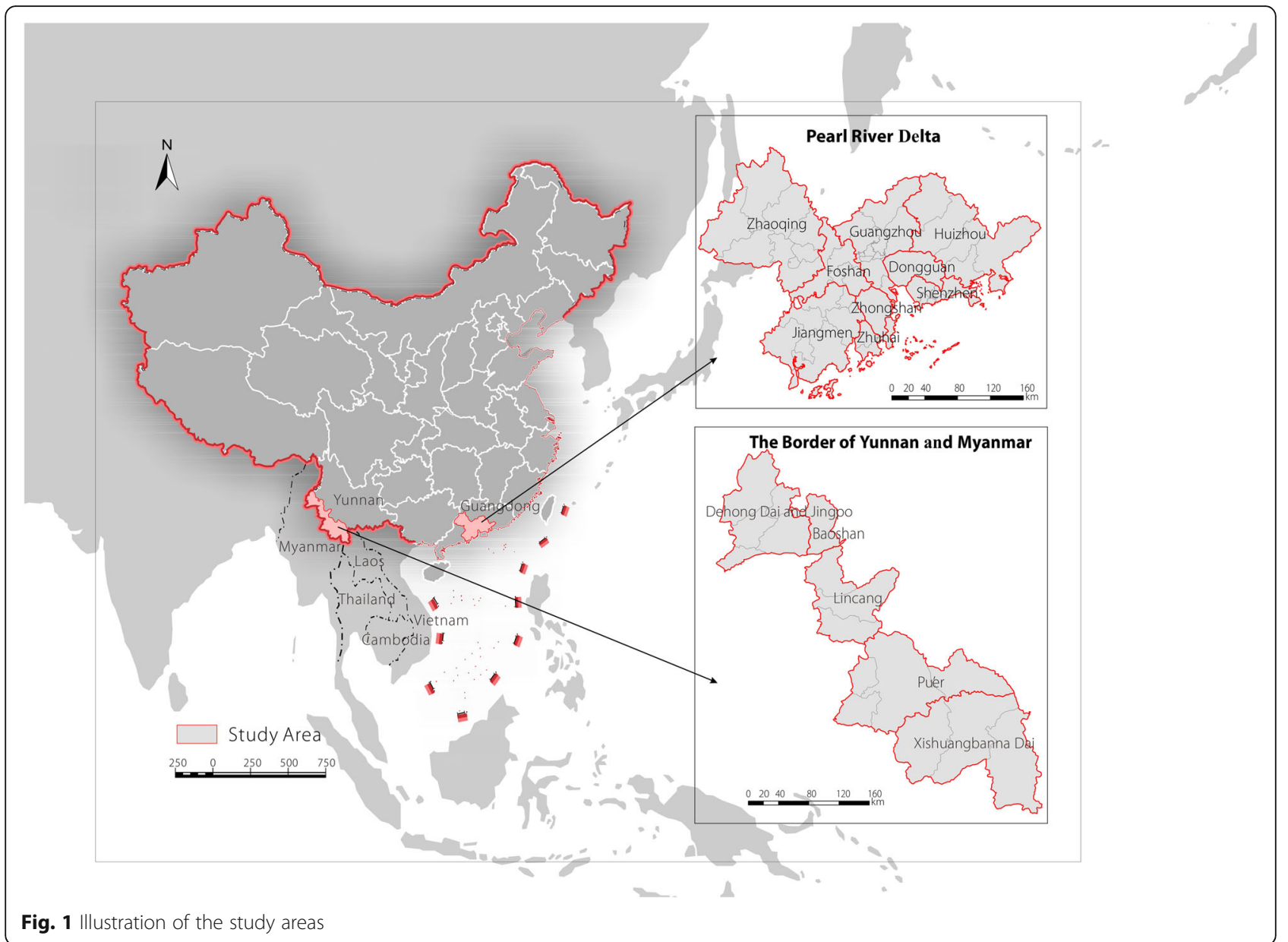

\section{Environmental and socioeconomic data}

In accordance with previous studies [21, 27-29], this study selected four environmental factors (mean temperature [Temp], mean relative humidity [Hum], mean precipitation [Pre], and normalized difference vegetation index [NDVI]) that temporally correspond to the epidemic data (from April to November). In addition, four socioeconomic variables in 2010 (land use data, population size, road density, and GDP) were obtained to reflect the regional social conditions. All variables (see Table 1 for details on data processing) were calculated from original data of $1 \mathrm{~km}^{2}$ resolution, and the spatial distribution of these factors is illustrated in Figs. 2 and 3.

\section{Research unit}

Basic geographic units, such as districts, counties, towns, and streets, are frequently altered by the constant changes in administrative divisions in epidemiological studies. However, this phenomenon can be effectively avoided by creating regular spatial grids [30]. A spatial autocorrelation analysis is often used to reflect the spatial aggregation of a feature in the region. In this study, a series of spatial grids $(1 \times 1$ $\mathrm{km}-14 \times 14 \mathrm{~km})$ was created, and the optimal grid of the DF spatial pattern in each region was selected based on Moran's $I$ [31]. Moran's $I$ is expressed by Eq. (1) as follows:

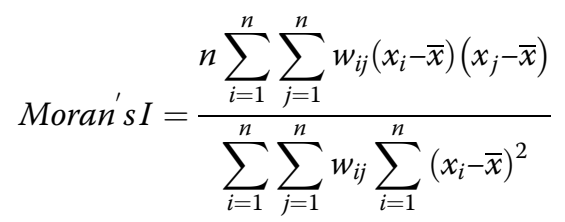

where $n$ is the number of grids in the study area, $x_{i}$ and $x_{j}$ represent the number of DF cases in grids $i$ and $j$, respectively, and $w_{i j}$ is the matrix of spatial weight. Moran's $I$ is generally tested by the $Z$-score/ $P$-value, and the value varies from -1 to 1 . A higher Moran's $I$ (larger $Z$-score and proper $P$-value) indicates greater similarity among attributes between adjacent spatial grids [32], which reveals that the DF epidemic is clustered in the region, whereas a low negative value indicates dissimilarity between adjacent grids and shows that the DF epidemic is discretely 
Table 1 Data sources and processing of environmental and socioeconomic factors

\begin{tabular}{|c|c|c|}
\hline Variables/description & Data processing & Data source \\
\hline $\begin{array}{l}\text { Mean } \\
\text { temperature }\end{array}$ & $\begin{array}{l}\text { Mean temperature from } 2010 \text { to } 2014 \text { (April-November) } \\
\text { in each grid }\end{array}$ & $\begin{array}{l}\text { China Meteorological Data Service Center } \\
\text { (CMDC, http://data.cma.cn/) }\end{array}$ \\
\hline Mean precipitation & $\begin{array}{l}\text { Mean precipitation from } 2010 \text { to } 2014 \text { (April-November) } \\
\text { in each grid }\end{array}$ & \\
\hline Mean relative humidity & $\begin{array}{l}\text { Mean relative humidity from } 2010 \text { to } 2014 \text { (April-November) } \\
\text { in each grid }\end{array}$ & \\
\hline Vegetation index & $\begin{array}{l}\text { Mean normalized difference vegetation index (NDVI) from } \\
2010 \text { to } 2014 \text { (April-November) in each grid }\end{array}$ & https://ladsweb.modaps.eosdis.nasa.gov/ \\
\hline Land use & $\begin{array}{l}\text { According to the land use coverage classification system of } \\
\text { the Data Center for RESDC, land use data in } 2010 \text { were divided } \\
\text { into cultivated land, forest land, grass land, water areas, urban } \\
\text { land, rural residential areas, other construction land, and } \\
\text { unused land. Statistics on the proportion of various land } \\
\text { use areas in the grid }\end{array}$ & $\begin{array}{l}\text { Resource and Environmental Science Data Center } \\
\text { of the Chinese Academy of Sciences } \\
\text { (RESDC, www.resdc.cn) }\end{array}$ \\
\hline Population size & $\begin{array}{l}\text { Summing the population (persons) for each grid based on the } \\
2010 \text { population density data }\end{array}$ & \\
\hline $\begin{array}{l}\text { Economic } \\
\text { conditions }\end{array}$ & $\begin{array}{l}\text { Summing the gross domestic product (GDP) values (RMB) for } \\
\text { each grid based on the } 2010 \text { GDP data }\end{array}$ & \\
\hline $\begin{array}{l}\text { Road } \\
\text { density }\end{array}$ & $\begin{array}{l}\text { Summing the road density }\left(\mathrm{km} / \mathrm{km}^{2}\right) \text { for each grid based on } \\
\text { the road network data in } 2010\end{array}$ & OpenStreetMap (http://download.geofabrik.de/) \\
\hline
\end{tabular}

distributed in the region [33]. In this study, Moran's $I$ and $Z$-scores of the DF cases with different grid sizes were used to assess the optimal grid scales of the regional DF epidemic. Spatial autocorrelation analysis above was performed using ArcGIS 10.2 (ESRI, Redlands, CA, USA).

\section{Statistical analysis}

The GAM is a semiparametric model extended from the generalized linear model $[34,35]$. It can provide both linear and nonlinear fitting to variables, and it has been widely used in infectious epidemiology, such as for DF, in recent years $[23,35,36]$. The model automatically selects the appropriate polynomial by establishing the smoothing function of the independent variable and identifies and estimates the nonlinear optimality of the model from data.

$$
g(\mu)=\beta_{0}+\sum \beta_{i}\left(X_{i}\right)+\sum S_{i}\left(X_{i}\right)
$$

In Eq. (2), $g(\mu)$ denotes a link function that can select the corresponding link function according to the different statistical distributions of dependent variables. Consistent with previous studies, the distribution of DF cases in this study fits a Poisson distribution [23]. Thus, the corresponding link function for the GAM model is $\log (\mathrm{y})$. The variable $\mathrm{y}$ refers to the number of local DF cases in the grid from 2010 to $2014(\log (D F$ case $)), \beta_{0}$ is a constant term, $\beta_{i}\left(X_{i}\right)$ represents the linear fitting function, and $\mathrm{Si}(\mathrm{Xi})$ represents the nonlinear fitting function. The independent variable $X_{i}$ represents the 12 variables (ratio of land use area [cultivated land, forest land, water area, rural residential land, and urban land], population density [Pop], road density [Road], NDVI, GDP, Hum, Temp and Pre) under the optimal grid.

The first step is to build the single factor model by using the spline smoothing function of the GAM, and then the goodness of fit of single factors is statistically analyzed. Next, the variables that did not pass the significance test in the single factor analysis are removed. Then, variables with strong collinearity are sorted into groups, and one variable in each group and other variables without strong collinearity are selected to build the GAM until all permutations and combinations are considered. Finally, the optimal GAM is selected according to the Akaike information criterion (AIC), with a better model corresponding to smaller AIC values [21]. The spatial data processing was completed in ArcGIS 10.2 software, and all the statistical analyses were performed using the statistical software R 3.0.3 (Lucent Technologies, Jasmine Mountain, USA) with the mgcv library.

\section{Results}

\section{Temporal and spatial distribution of DF}

According to the China Notifiable Disease Surveillance System, 49290 local DF cases occurred in China between 2010 and 2014, with those in the PRD and BYM accounting for $97.06 \%$. Figure 4 shows that the DF epidemic had obvious seasonal characteristics. The epidemic was mainly concentrated in July to November, accounting for $99.95 \%$ of the annual cases, and reached the peak incidence from August to October. In these 5 years, there were 46422 and 1419 local DF cases in the PRD and BYM, respectively, showing an increasing trend 


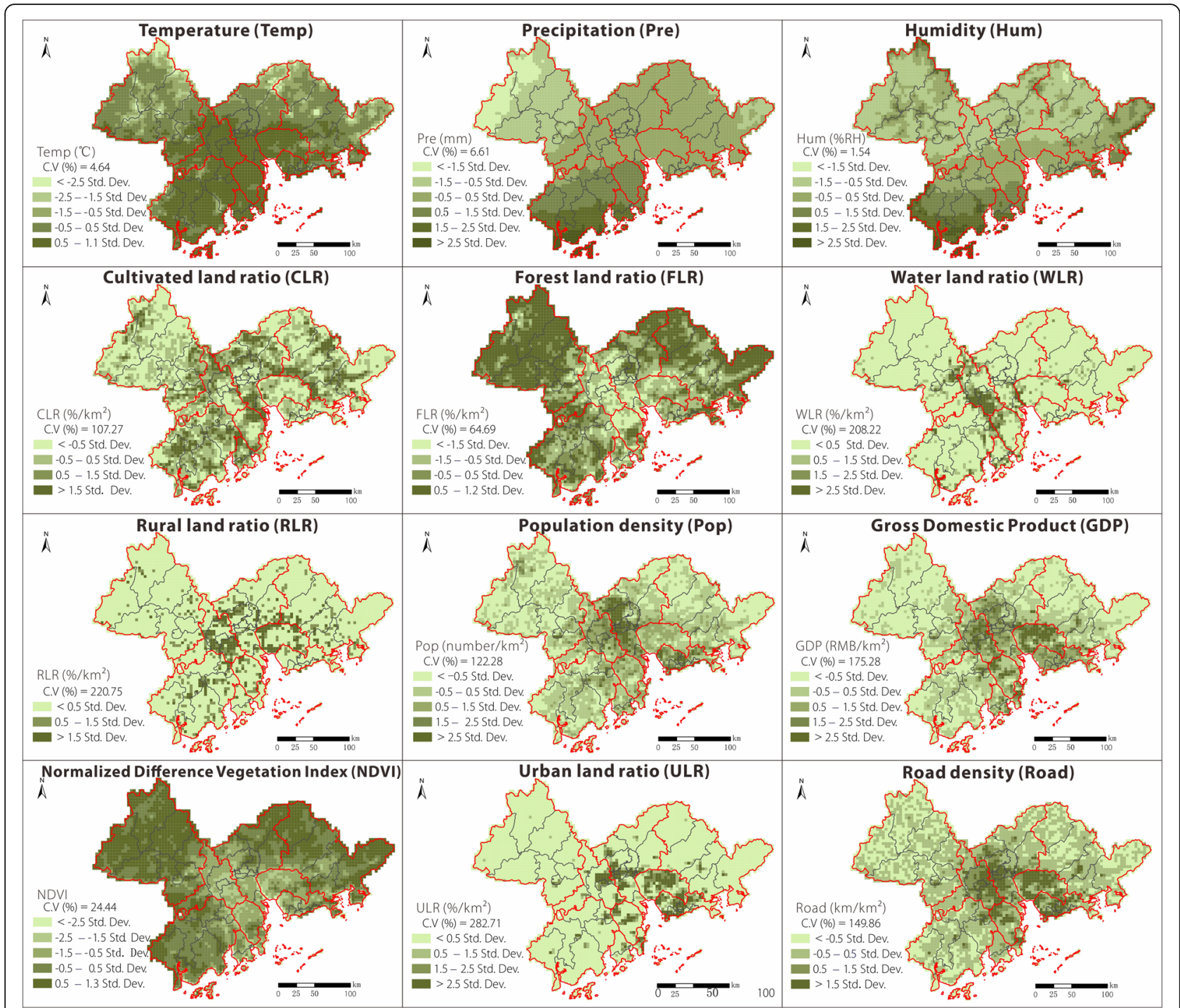

Fig. 2 Spatial distributions of environmental and socioeconomic factors in the Pearl River Delta; grid scale of $4 \mathrm{~km} \times 4 \mathrm{~km}$

in successive years. From 2010 to 2012, there were fewer than 500 local cases, and the number of cases in 2013 and 2014 surged to 4000 and 40000 , respectively, with the BYM exhibiting a large-scale epidemic in 2013.

Table 2 lists the Moran's $I$ values of the DF cases at different grid scales in the PRD and BYM, which were calculated by Eq. (1). The PRD showed better clustering of DF epidemic cases at the $4 \mathrm{~km} \times 4 \mathrm{~km}$ grid scale, whereas the BYM showed better clustering at the $3 \mathrm{~km} \times 3 \mathrm{~km}$ scale. Furthermore, all Moran's $I$ values in the PRD were greater than those in the BYM, which indicated that the DF epidemic of the PRD was highly aggregated, while that of the BYM was relatively decentralized. In terms of the spatial distribution mapped in Fig. 5, the DF cases in the PRD presented an aggregative distribution cantered in the Guangzhou-Foshan region, whereas cases in the
BYM were mainly concentrated in Dehong and Xishuangbanna Prefectures. These results demonstrate that DF cases in the BYM and the PRD showed significant characteristics of spatial aggregation.

\section{GAM fitting}

Pearson correlation coefficient analysis (see Additional files 2 and 3) and variable collinearity analysis (see Additional file 4) show that strong collinearity occurred in both the PRD (among the urban land ratio [ULR], road, and GDP; among Pop, Road, and GDP; and between the forest land ratio and NDVI) and the BYM (among Pop, ULR, and GDP; and between Hum and Pre). In each region, the model that passed the collinear diagnosis with the lowest AIC value was used as the optimal model for the DF epidemic to avoid over fitting of the model (see Additional file 4). Thus, the optimal GAM of 


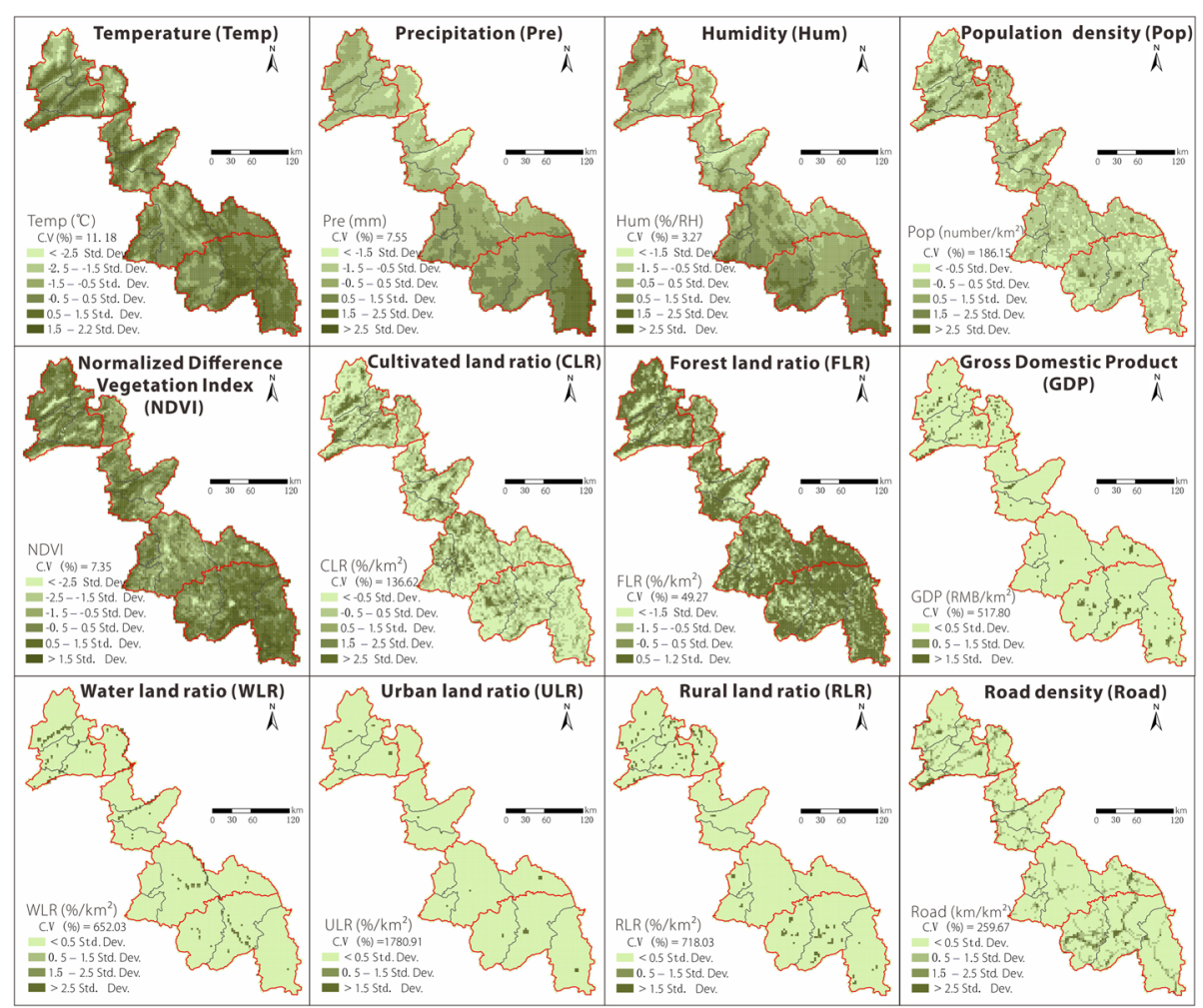

Fig. 3 Spatial distributions of the environmental and socioeconomic factors in the Border of Yunnan and Myanmar; grid scale of $3 \mathrm{~km} \times 3 \mathrm{~km}$

the DF epidemic in the PRD consisted of five variables (Pop, ULR, NDVI, Hum, and Temp), and that in the BYM consisted of six variables (ULR, Road, NDVI, Temp, water land ratio [WLR], and Pre). The total variance of the GAM for the DF epidemic was $83.4 \%\left(R^{2}=0.834, P R D\right)$ and
97.3\% $\left(R^{2}=0.973, B Y M\right)$, which shows that the GAM fit the regional differences of the epidemic well.

Regarding the variance explained by the single factor in the optimal GAM (see Additional file 4), socioeconomic factors explained more of the variance $(>54 \%)$ than

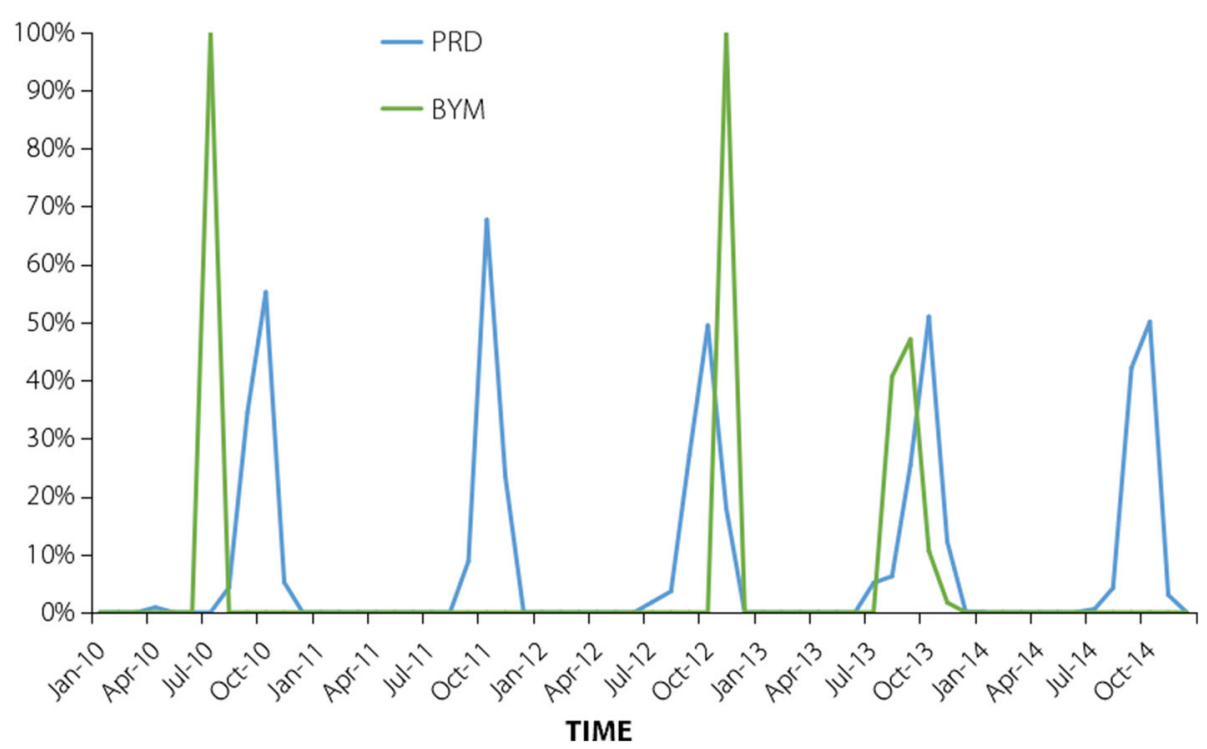

Fig. 4 Ratio of monthly local cases to the total annual local cases in 2010-2014; PRD: Pearl River Delta; BYM: Border of Yunnan and Myanmar 
Table 2 Spatial autocorrelation analysis of dengue fever cases in the Pearl River Delta and the Border of Yunnan and Myanmar from 2010 to 2014

\begin{tabular}{|c|c|c|c|c|c|c|c|c|c|c|c|c|c|c|c|}
\hline & $\begin{array}{l}\text { Gridded } \\
\text { Scales }\end{array}$ & $1 \mathrm{~km}$ & $2 \mathrm{~km}$ & $3 \mathrm{~km}$ & $4 \mathrm{~km}$ & $5 \mathrm{~km}$ & $6 \mathrm{~km}$ & $7 \mathrm{~km}$ & $8 \mathrm{~km}$ & $9 \mathrm{~km}$ & $10 \mathrm{~km}$ & $11 \mathrm{~km}$ & $12 \mathrm{~km}$ & $13 \mathrm{~km}$ & $14 \mathrm{~km}$ \\
\hline \multirow[t]{3}{*}{ Pearl River Delta } & Moran's I & 0.59 & 0.73 & 0.73 & 0.77 & 0.73 & 0.67 & 0.65 & 0.47 & 0.57 & 0.54 & 0.60 & 0.31 & 0.40 & 0.46 \\
\hline & Z-score & 417.16 & 206.80 & 119.04 & 69.81 & 57.31 & 45.01 & 40.31 & 35.73 & 29.69 & 26.46 & 23.15 & 20.76 & 21.28 & 18.08 \\
\hline & $P$-value & $<0.01$ & $<0.01$ & $<0.01$ & $<0.01$ & $<0.01$ & $<0.01$ & $<0.01$ & $<0.01$ & $<0.01$ & $<0.01$ & $<0.01$ & $<0.01$ & $<0.01$ & $<0.01$ \\
\hline \multirow{3}{*}{$\begin{array}{l}\text { Border of } \\
\text { Yunnan and } \\
\text { Myanmar }\end{array}$} & Moran's / & 0.15 & 0.23 & 0.31 & 0.18 & 0.27 & 0.27 & 0.11 & 0.01 & 0.31 & 0.27 & 0.22 & 0.27 & 0.01 & 0.12 \\
\hline & Z-score & 90.17 & 54.62 & 47.75 & 20.57 & 26.88 & 22.26 & 20.79 & 3.74 & 16.59 & 13.63 & 11.98 & 12.11 & 2.10 & 10.44 \\
\hline & $P$-value & $<0.01$ & $<0.01$ & $<0.01$ & $<0.01$ & $<0.01$ & $<0.01$ & $<0.01$ & $<0.01$ & $<0.01$ & $<0.01$ & $<0.01$ & $<0.01$ & $<0.05$ & $<0.0$ \\
\hline
\end{tabular}

boldface: dengue fever cases showed significant characteristics on this grid scale

environmental factors $(<54 \%)$. Among the socioeconomic factors, Pop and ULR explained the greatest variance in the DF epidemic in the PRD, whereas the ULR, followed by road density, explained the greatest variance in the DF epidemic in the BYM. For environmental conditions, NDVI ranked first in the two regions, followed by Hum-Temp (PRD) and Temp-WLR-Pre (BYM). These results show that the factors affecting the DF epidemic were generally similar but presented slight differences between the PRD and BYM.

\section{Comparison of the main DF factors in the two regions}

As shown in Fig. 6, the nonlinear characteristics between the DF epidemic and the independent variables were obvious. In terms of the PRD, the DF epidemic was more serious in areas (grids) with a higher socioeconomic status, especially in the areas with $440<$ Pop $<3500$ (Fig. 6A1) and ULR $>0.4$ (Fig. 6A2), while the DF epidemic tended to be stable in areas with a Pop $>3500$ (Fig. 6A1). Compared with Pop and ULR, the nonlinear characteristics between environmental factors and the DF epidemic were more obvious. Among the factors, an ' $M$ ' relationship was observed between NDVI and the DF epidemic (Fig. 6A3), and the DF epidemic was serious when the NDVI was between 0.17 and 0.76 , especially from 0.17 to 0.4 . Similar to the NDVI, Hum also had an ' $M$ ' relationship with DF (Fig. 6A4), and the DF epidemic was relatively serious when Hum in the region was $79.2-82.5 \%$. In contrast to the pattern observed for the above factors, the DF epidemic showed a clear "break" when the

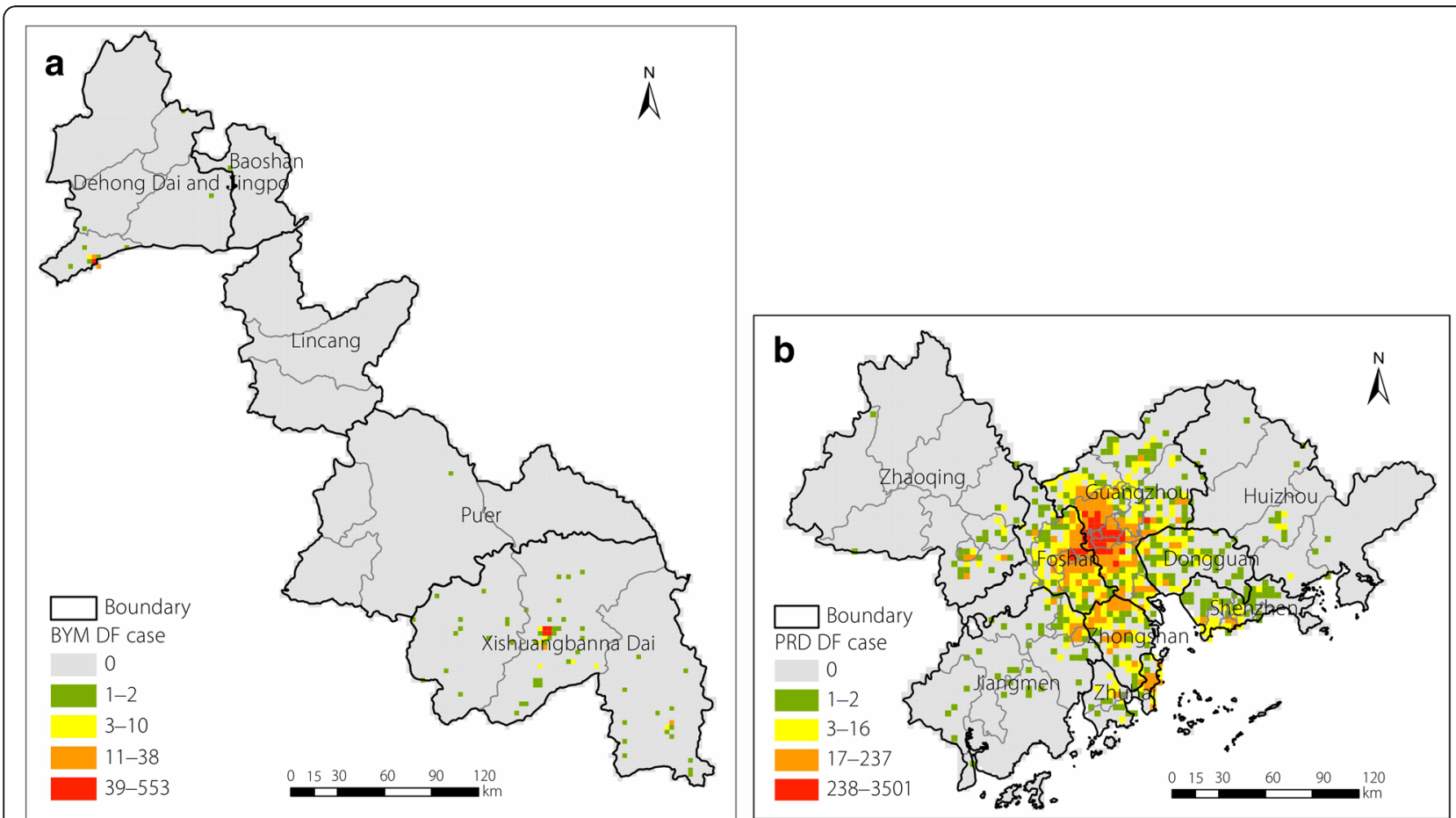

Fig. 5 Spatial distribution of DF in the PRD and BYM; a BYM grids of $3 \mathrm{~km} \times 3 \mathrm{~km}$, and $\mathbf{b}$ PRD grids of $4 \mathrm{~km} \times 4 \mathrm{~km}$; DF: dengue fever; PRD: Pearl River Delta; BYM: Border of Yunnan and Myanmar 

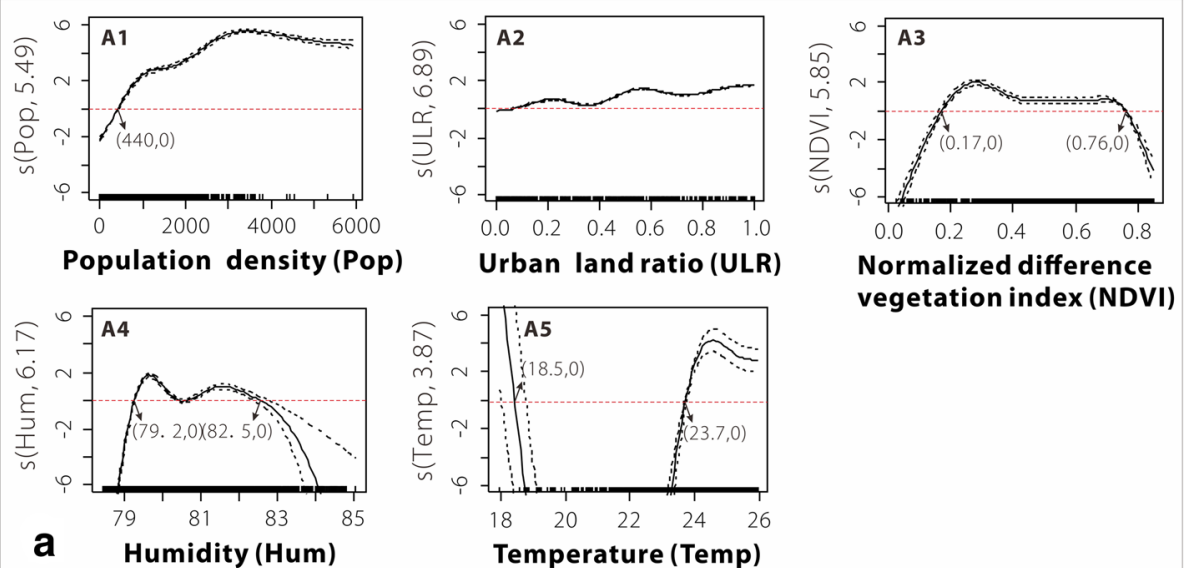

vegetation index (NDVI)
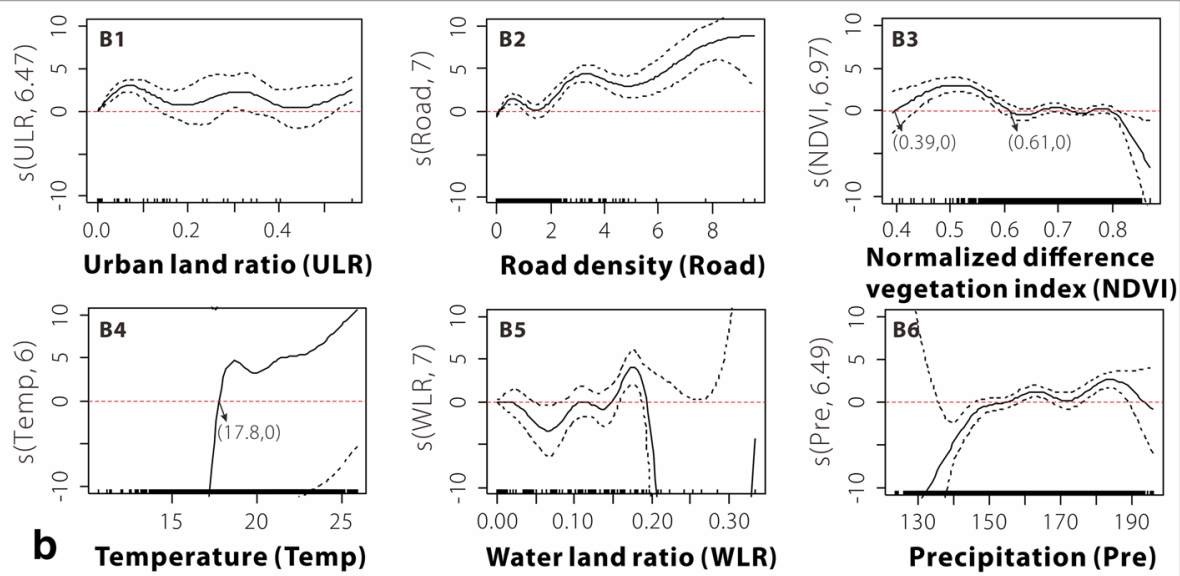

Fig. 6 Generalized additive models-estimated relationships between dengue fever cases and influencing factors; a Pearl River Delta (PRD), A1: Population density (Pop), A2: Urban land ratio (ULR), A3: Normalized difference vegetation index (NDVI), A4: Humidity (Hum), A5: Temperature (Temp), b Border of Yunnan and Myanmar (BYM), B1, Urban land ratio (ULR), B2: Road density (Road), B3: Normalized difference vegetation index (NDVI), B4: Temperature (Temp), B5: Water land ratio (WLR), B6: Precipitation (Pre). The solid line shows the smooth fitting curve for the logarithm of dengue fever cases. The dashed line represents the $95 \%$ confidence intervals. The $x$-axis represents the actual values of the independent variables. The $y$-axis indicates the logarithm of dengue fever cases fitting values. Edf represents the estimated degrees of freedom. The $y$-axis is labelled $s(a$, edf), where a indicates the name of the variables and edf represents the estimated degrees of freedom of the smooth function, which is used to represent its relationship with dengue fever cases

Temp of the region was $19-23^{\circ} \mathrm{C}$ (Fig. 6A5), while the DF epidemic was more severe when Temp was below $18.5^{\circ} \mathrm{C}$ (limited distribution) or above $23.7^{\circ} \mathrm{C}$ (wider distribution).

Compared with the PRD, the DF epidemic in the BYM was relatively clustered in urban areas and showed gentle fluctuations with increases in ULR (Fig. 6B1). The ULR of the areas with relatively serious DF epidemics was approximately 0.07 and 0.3 , and DF epidemics showed a wave rise as the road density increased (Fig. 6B2). Although the DF epidemic and the NDVI (ranking first among environmental factors) also showed an ' $M$ ' relationship in the BYM (Fig. 6B3), the NDVI value in relatively severe epidemic areas was $0.39-0.61$. In addition, the DF epidemic was relatively serious in the BYM where the average Temp was higher than $17.8^{\circ} \mathrm{C}$ (Fig. 6B4), the WLR was between 0.15 and 0.20 (Fig. 6B5), and the Pre was approximately $180 \mathrm{~mm}$ (Fig. 6B6). In general, the main factors (socioeconomic and environmental factors) and their nonlinear relationships with DF epidemics in the PRD and BYM were significantly different.

In terms of spatial distribution, Guangzhou-Foshan, as well as Dongguan and Shenzhen, were areas with serious DF epidemics in the PRD (Fig. 7a). These areas appeared to have high ULR $(>0.4)$, high Pop $(>430)$, and moderate NDVI $(0.17<$ NDVI $<0.76)$. In addition, DF epidemics were more serious if the Hum was moderate (approximately $79.5 \%$ or $81.5 \%$ ) and the Temp was higher than $23.7^{\circ} \mathrm{C}$. In comparison, DF epidemics in the BYM were relatively scattered throughout the Dehong and Xishuangbanna Prefectures (Fig. 7b). These areas have ULRs ranging from 0 to 0.5 , developed road networks (road density $\left.>2 \mathrm{~km} / \mathrm{km}^{2}\right)$, and moderate NDVIs $(0.39<\mathrm{NDVI}$ $<0.61)$. DF epidemics were more acute in areas with Temp 


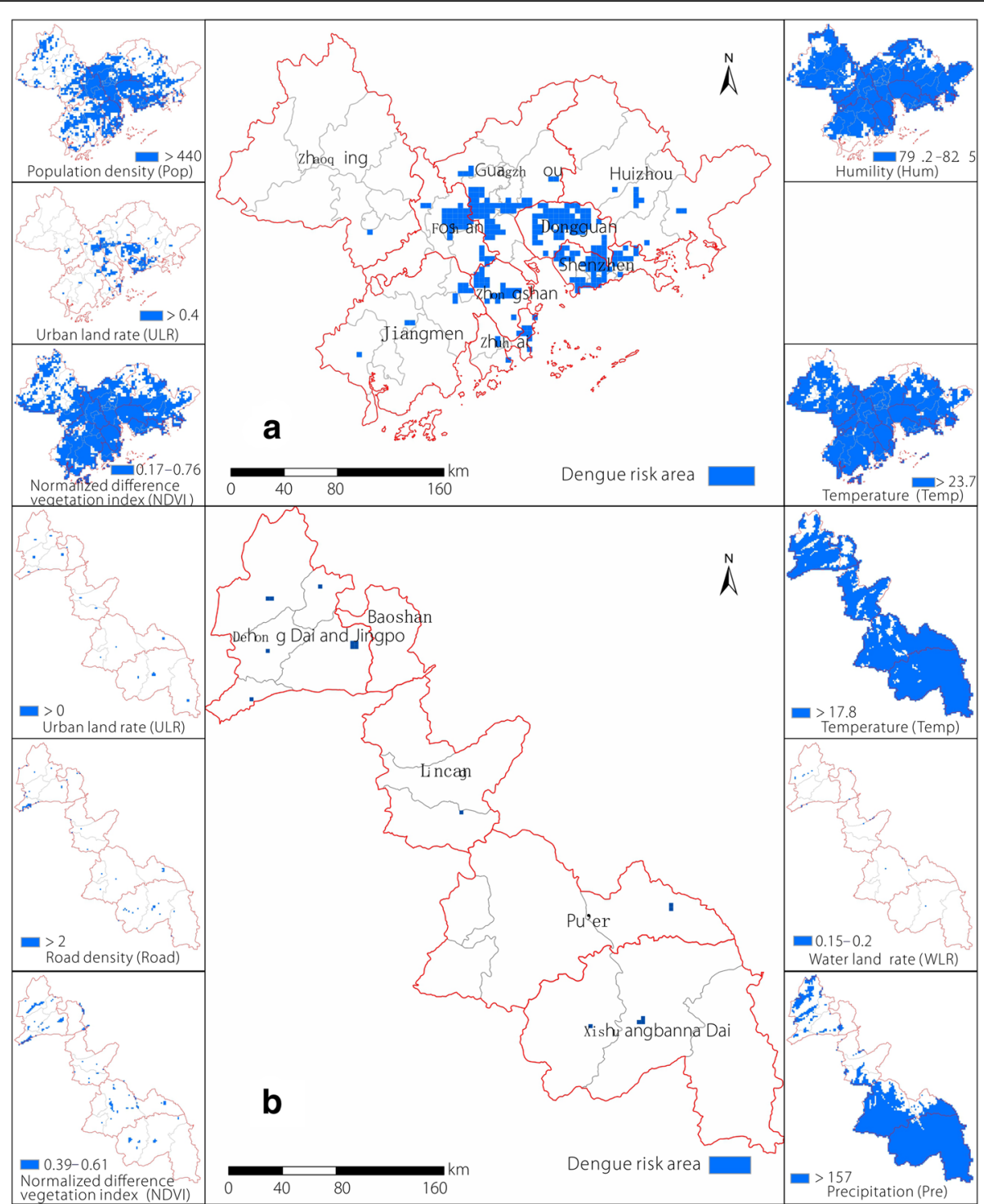

Fig. 7 Dengue fever risk areas derived from the generalized additive models fitting results; a Pearl River Delta (PRD), and $\mathbf{b}$ Border of Yunnan and Myanmar (BYM)

$>17.8^{\circ} \mathrm{C}$, Pre at moderate levels $(170-190 \mathrm{~mm})$, or water bodies (ratio of approximately $0.15-0.2$ ).

\section{Discussion}

In this study, we used the GAM to analyse and compare the main factors that affect the differences between DF epidemics in the PRD and BYM on the grid scale. Strengthening our understanding of the spatial-temporal patterns and differences between the influencing factors of DF epidemics in China's typical DF epidemic areas is of considerable significance because such data can improve our ability to prevent and control the DF risk in high-incidence areas.

The epidemic characteristics of the PRD and BYM have quite a few similarities in environmental and socioeconomic factors. Regarding environmental conditions, previous studies have shown that suitable meteorological conditions (Temp at $20-30^{\circ} \mathrm{C}$ and Hum of $75 \%$ were conducive to mosquito breeding and reproduction $[37,38]$, thereby promoting dengue virus activity and increasing the risk of DF epidemics and transmission [39, 40]. According to the basic data of this study, the monthly mean Temp in the PRD and BYM is between $10{ }^{\circ} \mathrm{C}$ and $27^{\circ} \mathrm{C}$ from April to November, the monthly mean Pre is $0-360 \mathrm{~mm}$, and the relative Hum is $63-84 \%$. These warm and humid weather conditions are conducive to DF transmission. In addition, the vegetation conditions in these two regions are good (the average NDVI is 0.57 to 0.81 ). Higher or lower NDVI values will reduce the risk of DF [41, 42]. High NDVI 
values generally indicate sparsely populated areas that are unable to meet the conditions of human and mosquito contact for DF. The appropriate vegetation conditions provide not only excellent conditions for the breeding and activity of mosquitoes (Aedes albopictus is primarily observed in the PRD, and Aedes aegypti is primarily observed in the BYM) [43] but also cool locations in summer for humans, thereby increasing the probability of contact between humans and mosquitoes and promoting DF epidemic risk [44]. The warm and humid environmental conditions in the BYM and PRD are suitable for mosquito breeding and activity, which is an important reason for the rapid and widespread prevalence of DF and presents the first similarity of DF epidemics in these two regions.

As for the second similarity of the characteristics of DF epidemic in these two regions, Ren et al. considered that socioeconomic factors might play a significant role in DF epidemics in cases where environmental factors were suitable and differed slightly in regional [13]. This finding is consistent with our results. Although the socioeconomic status (Pop, ULR, and Road) of the PRD is significantly greater than that of the BYM, these two regions present similar differences in development, and this finding can also be confirmed that the coefficient of variation of socioeconomic factors is significantly higher than that of environmental factors. In addition, Zhu et al. noted that a certain degree of population aggregation was an important condition for DF transmission and prevalence [45]. The population is relatively clustered in towns of the BYM (such as Dehong and Xishuangbanna) and the highly urbanized regions of the PRD (such as Guangzhou-Foshan). Therefore, this condition gives a reasonable explanation for the relatively serious epidemic in these areas. In recent years, the PRD has implemented active prevention and control measures, such as water retention and mosquito prevention, and DF epidemics have been effectively controlled. Therefore, based on the similarities between the two regions regarding the spatial patterns and main influencing factors underlying DF epidemics, we suggest that the BYM can draw on the experience of the PRD when formulating DF epidemic prevention and control strategies at the regional level.

Compared with the similarities listed above, the differences in the DF epidemic characteristics between the PRD and the BYM should be further investigated. Regarding the socioeconomic factors, previous studies have shown that a higher ULR corresponded to a larger population, while developed transport networks increased the mobility of people in the region [23, 24, 46, 47]. The extent and concentration of DF epidemics in the PRD are higher than those in the BYM, which is closely related to the higher Pop, higher ULR, uniform road network distribution, and stronger population mobility. The ULR of the BYM is high only in the central town areas of Dehong and Xishuangbanna (but still lower than that of the PRD), as are Pop and Road. The accessibility of roads throughout the region is not as good as that in the PRD, which can also explain why DF epidemics in the PRD are significantly greater than those in the BYM.

As for the regional environmental factors, the overall vegetation coverage in the BYM is relatively high. Therefore, the NDVI fitting curve of the more serious epidemic areas is shifted to the left compared with that in the PRD. Furthermore, the terrain within the BYM is complex, including large numbers of mountains and valleys. Therefore, regional differences and vertical changes in climate are obvious [48], which is different from the decreasing trend of temperature and precipitation in the PRD from south to north and from coast to inland [49]. The coefficient of variation of the environmental factors in the BYM is also slightly higher than that in the PRD. Meanwhile, the risk of DF tends to be higher in Dehong and Xishuangbanna Prefectures due to their higher annual average Temp and Pre, thus providing a favorable living environment for the Aedes mosquito, which is widely distributed throughout Ruili City (Dehong Prefecture) and Xishuangbanna Prefecture [50].

As for imported cases of DF, the BYM has several ports through which DF is mainly imported by "ground", including Jinghong Port, Simao Waterway Port, and the busiest port of Rili to Myanmar. These wide ranges of trade ports promote local economic development but also increase the risk of imported DF cases because DF is highly prevalent in areas adjacent to Laos and Myanmar. In addition, Wang et al. confirmed that the local DF epidemic in the BYM in 2013 was caused by imported cases from the neighbouring countries of Southeast Asia [51]. The developed economy of the PRD results in the majority of imported DF cases being brought by relatively long-distance commercial travel. Meanwhile, as one of the most densely populated areas in China, more than one million migrant workers travel to the PRD each year. These people live in crowded and poor sanitary conditions and less rectified living areas, which are conditions conducive to human-mosquito contact [23, 46]. The BYM and PRD are both important ports of land and sea-air entry and exit in China, and they are popular locations for tourists and migrant workers in China. If DF epidemics develop explosive outbreaks in both regions without being effectively controlled, they will spread to adjacent inland areas, which will cause serious impacts to the life and health of people in inland areas. Therefore, the health departments must 
consider the spatial differentiation characteristics of the main factors affecting DF epidemics in the region and utilize these data to formulate more specific prevention and control strategies.

Several limitations are worth noting. (1) The difference in the severity of the two regional epidemics leads to a significant difference in the confidence interval of the fitting curve, although it does not affect the research paradigm. (2) The effects of mosquito vector and control measures have not been sufficiently considered, and these data should be included in our future work. (3) The spatial correlation between DF epidemics and various influencing factors has not been properly considered in the model, and models such as the GWR, which can consider such spatial correlations, should be included in future studies. (4) This study used the number of DF cases from 2010 to 2014 as the dependent variable, without carefully analysing the lagging effect and temporal effect between DF and its influencing factors.

\section{Conclusions}

The environmental and socioeconomic factors in the PRD and BYM may affect the spatial-temporal differentiation of DF epidemics, and the influencing mechanisms have their own regional characteristics. The differences in socioeconomic factors are more obvious in cases where environmental factors are suitable and differ slightly throughout areas. This study has improved our understanding of the spatial distribution of DF epidemics and their influencing factors in typical regions of China. We suggest that the epidemic prevention and control strategies for the BYM should be developed in reference to those for the PRD, combined with the characteristics of the main factors influencing the regional epidemic to effectively strengthen the prevention and control measures for DF epidemics.

\section{Additional files}

Additional file 1: Multilingual abstracts in the five official working languages of the United Nations. (PDF $225 \mathrm{~kb}$ )

Additional file 2: Pearson correlation analysis of variables in the PRD. (DOCX $19 \mathrm{~kb}$ )

Additional file 3: Pearson correlation analysis of variables in the BYM. (DOCX $18 \mathrm{~kb}$ )

Additional file 4: Statistical characteristics of variables and GAM fitting results. (XLSX $14 \mathrm{~kb})$

\section{Abbreviations}

AIC: Akaike Information Criterion; BYM: The Border of Yunnan and Myanmar; CLR: Cultivated land ratio; DF: Dengue fever; ESRI: Environmental Systems Research Institute; FLR: Forest land ratio; GAM: Generalized additive model; GDP: Gross domestic product; Hum: Humidity; NDVI: Normalized difference vegetation index; Pop: Population density; PRD: Pearl River Delta of China;
Pre: Precipitation; RLR: Rural land ratio; Road: Road density; Temp: Temperature; ULR: Urban land ratio; USA: United States of America; WLR: Water land ratio

\section{Acknowledgements}

The authors would like to thank Qiao-Xuan Li for his advice and contribution on spatial-epidemiological aspects.

\section{Funding}

This work was supported by the National Natural Science Foundation of China (No. 41571158), the National Key Research and Development Program of China (No. 2016YFC1302602), the National Key Research and Development Program of China (No. 2016YFC1201305), the Shanghai Municipal Commission of Health and Family Planning (No. 15GWZK0201), the Innovation Project of LREIS (No. O8R8B6A0YA), the Fundamental Research Funds for the Central Universities in China (East China Normal University).

Availability of data and materials

All data generated or analysed during this study and supporting the conclusions of this article are included in the article.

\section{Authors' contributions}

$\mathrm{H}-\mathrm{YR}$ and R-HS conceived and designed the experimental concept. LL helped collect data. LZ analysed the data and drafted the paper. H-YR and R-HS revised the manuscript. All authors read and approved the final manuscript.

Ethics approval and consent to participate Not applicable.

\section{Consent for publication}

Not applicable.

\section{Competing interests}

The authors declare that they have no competing interests.

\section{Author details}

${ }^{1}$ Key Laboratory of Geographic Information Science, Ministry of Education, East China Normal University, Shanghai, China. ${ }^{2}$ State Key Laboratory of Resources and Environmental Information System, Institute of Geographic Sciences and Natural Resources Research, Chinese Academy of Sciences, Beijing, China. ${ }^{3}$ School of Geographic Sciences, East China Normal University, Shanghai, China. ${ }^{4}$ Joint Laboratory for Environmental Remote Sensing and Data Assimilation, East China Normal University and Institute of Remote Sensing and Digital Earth, Chinese Academy of Sciences, Shanghai, China. ${ }^{5}$ Department of Vector Biology and Control, Chinese Center for Disease Control and Prevention, Natural Institute for Communicable Disease Control and Prevention, Beijing, China.

Received: 20 April 2018 Accepted: 12 March 2019

Published online: 28 March 2019

References

1. Wu JY, Lun ZR, James AA, Chen XG. Review: dengue fever in mainland China. Am J Trop Med Hyg. 2010;83:664-71.

2. Guo C, Zhou Z, Wen Z, Liu Y, Zeng C, Xiao D, et al. Global epidemiology of dengue outbreaks in 1990-2015: a systematic review and meta-analysis. Front Cell Infect Microbiol. 2017;7:317

3. Rodriguez-Morales AJ, Ruiz P, Tabares J, Ossa CA, Yepes-Echeverry MC Ramirez-Jaramillo $\mathrm{V}$, et al. Mapping the ecoepidemiology of Zika virus infection in urban and rural areas of Pereira, Risaralda, Colombia, 2015-2016: implications for public health and travel medicine. Travel Med Infect Dis. 2017:18:57-66

4. Ren H, Ning W, Lu L, Zhuang D, Liu Q. Characterization of dengue epidemics in mainland China over the past decade. J Infect Dev Ctries. 2015:9:970-6

5. $\quad$ Sang SW, Wang SS, Lu L, Bi P, Lv M, Liu QY. The epidemiological characteristics and dynamic transmission of dengue in China, 2013. PLoS Negl Trop Dis. 2016;10:e0005095.

6. Li MT, Sun GQ, Yakob L, Zhu HP, Jin Z, Zhang WY. The driving force for 2014 dengue outbreak in Guangdong, China. PLoS One. 2016;11:e0166211. 
7. Wang J, Chen H, Huang M, Zhang Y, Xie J, Yan Y, et al. Epidemiological and etiological investigation of dengue fever in the Fujian province of China during 2004-2014. Sci China Life Sci. 2017;60:72-80.

8. Gao Z, Zhang Y, Yang Y, Xu M, Liao P, He W, et al. Dengue virus infections among blood donors in Guangxi of China, 2013-2014. Transfus Med. 2017; 28:236-42.

9. Zheng J, Han W, Jiang B, Ma W, Zhang Y. Infectious diseases and tropical cyclones in Southeast China. Int J Environ Res Public Health. 2017;14:494.

10. Huang XY, Ma HX, Wang HF, Du YH, Su J, Li XL, et al. Outbreak of dengue fever in Central China, 2013. Biomed Environ Sci. 2014;27:894-7.

11. Fan J, Lin H, Wang C, Bai L, Yang S, Chu C, et al. Identifying the highrisk areas and associated meteorological factors of dengue transmission in Guangdong Province, China from 2005 to 2011. Epidemiol Infect. 2014;142:634-43.

12. Liu C, Liu Q, Lin H, Xin B, Nie J. Spatial analysis of dengue fever in Guangdong Province, China, 2001-2006. Asia Pac J Public Health. 2014; 26:58-66.

13. Yu A, Luo Y, Yang Y, Yan H, Zhou H. Investigation of the prevalence of dengue fever in the port of Menglian, Yunnan Province. J Pathogen Biol. 2015;10:442-5

14. Yang M, Jiang J, Guo X, Wu C, Zhou H. Epidemiological analysis and investigation of dengue fever in Yunnan Province from 2009 to 2014. J Pathogen Biol. 2015:10:738-42.

15. Aguilera I, Eeftens M, Meier R, Ducret-Stich RE, Schindler C, Ineichen A, et al. Land use regression models for crustal and traffic-related PM2.5 constituents in four areas of the SAPALDIA study. Environ Res. 2015;140: 377-84.

16. Bhatt S, Gething PW, Brady OJ, Messina JP, Farlow AW, Moyes CL, et al. The global distribution and burden of dengue. Nature. 2013:496:504-7.

17. Hales S, de Wet N, Maindonald J, Woodward A. Potential effect of population and climate changes on global distribution of dengue fever: an empirical model. Lancet. 2002:360:830-4.

18. Chang $K$, Chen CD, Shih CM, Lee TC, Wu MT, Wu DC, et al. Time-lagging interplay effect and excess risk of meteorological/mosquito parameters and petrochemical gas explosion on dengue incidence. Sci Rep. 2016;6:35028.

19. Bai L, Morton LC, Liu Q. Climate change and mosquito-borne diseases in China: a review. Glob Health. 2013;9:10.

20. Cardoso-Leite R, Vilarinho AC, Novaes MC, Tonetto AF, Vilardi GC, GuillermoFerreira R. Recent and future environmental suitability to dengue fever in Brazil using species distribution model. Trans R Soc Trop Med Hyg. 2014; 108:99-104.

21. Bouzid M, Colon-Gonzalez FJ, Lung T, Lake IR, Hunter PR. Climate change and the emergence of vector-borne diseases in Europe: case study of dengue fever. BMC Public Health. 2014;14:781.

22. Ibarra AMS, Ryan SJ, Beltran E, Mejia R, Silva M, Munoz A. Dengue vector dynamics (Aedes aegypti) influenced by climate and social factors in Ecuador: implications for targeted control. PLoS One. 2013:8:e78263.

23. Qi X, Wang Y, Li Y, Meng Y, Chen Q, Ma J, et al. The effects of socioeconomic and environmental factors on the incidence of dengue fever in the Pearl River Delta, China, 2013. PLoS Negl Trop Dis. 2015;9: e0004159.

24. Li Q, Ren H, Zheng L, Cao W, Zhang A, Zhuang D, et al. Ecological niche modeling identifies fine-scale areas at high risk of dengue fever in the Pearl River Delta, China. Int J Environ Res Public Health. 2017;14:619.

25. Ren H, Zheng L, Li Q, Yuan W, Lu L. Exploring determinants of spatial variations in the dengue fever epidemic using geographically weighted regression model: a case study in the joint Guangzhou-Foshan area, China, 2014. Int J Environ Res Public Health. 2017;14:1518.

26. Liu DD, Guo SL, Chen XH, Shao QX. Analysis of trends of annual and seasonal precipitation from 1956 to 2000 in Guangdong Province, China. Hydrolog Sci J. 2012;57:358-69.

27. Braga C, Luna CF, Martelli CM, de Souza WV, Cordeiro MT, Alexander N, et al. Seroprevalence and risk factors for dengue infection in socioeconomically distinct areas of Recife, Brazil. Acta Trop. 2010;113:234-40.

28. Rubio-Palis Y, Perez-Ybarra LM, Infante-Ruiz M, Comach G, UrdanetaMarquez L. Influence of climatic variables on dengue cases and abundance of Aedes aegypti (Diptera: Culicidae) in Maracay, Venezuela. B Malariol Salud Amb. 2011;51:145-57.

29. Yi B, Xu D, Zhang Z, Zhang B, Xi Y, Fu J, et al. Study on the relationship between NDVI of NOAA-AVHRR image and the aedes density in Guangdong Province. J Fourth Mil Med Univ. 2003;24:1720-4.
30. Ren H, Xu D, Shi X, Xu J, Zhuang D, Yang G. Characterisation of gastric cancer and its relation to environmental factors: a case study in Shenqiu County, China. Int J Environ Health Res. 2016;26:1-10.

31. Ord JK, Getis A. Local spatial autocorrelation statistics: distributional issues and an application. Geogr Anal. 1995;27:286-306.

32. Boden G, Chen X, Polansky M. Disruption of circadian insulin secretion is associated with reduced glucose uptake in first-degree relatives of patients with type 2 diabetes. Diabetes. 1999:48:2182-8.

33. Mueller-Warrant GW, Whittaker GW, Young WC. GIS analysis of spatial clustering and temporal change in weeds of grass seed crops. Weed Sci. 2008:56:647-69.

34. Imai C, Hashizume M. A systematic review of methodology: time series regression analysis for environmental factors and infectious diseases. Trop Med Health. 2015;43:1-9.

35. Cheong YL, Burkart K, Leitao PJ, Lakes T. Assessing weather effects on dengue disease in Malaysia. Int J Environ Res Public Health. 2013;10: 6319-34.

36. Guisan A, Edwards TC, Hastie T. Generalized linear and generalized additive models in studies of species distributions: setting the scene. Ecol Model. 2002;157:89-100.

37. Wang CG, Jiang BF, Fan JC, Wang FR, Liu QY. A study of the dengue epidemic and meteorological factors in Guangzhou, China, by using a zeroinflated poisson regression model. Asia Pac J Public Health. 2014;26:48-57.

38. Hopp M, Foley JA. Worldwide fluctuations in dengue fever cases related to climate variability. Clim Res. 2003;25:85-94.

39. Bangs MJ, Larasati RP, Corwin AL, Wuryadi S. Climatic factors associated with epidemic dengue in Palembang, Indonesia: implications of short-term meteorological events on virus transmission. Southeast Asian J Trop Med Public Health. 2006;37:1103-16.

40. Azil AH, Long SA, Ritchie SA, Williams CR. The development of predictive tools for pre-emptive dengue vector control: a study of Aedes aegypti abundance and meteorological variables in North Queensland, Australia. Trop Med Int Health. 2010;15:1190-7.

41. Araujo RV, Albertini MR, Costa-da-Silva AL, Suesdek L, Franceschi NC, Bastos $\mathrm{NM}$, et al. Sao Paulo urban heat islands have a higher incidence of dengue than other urban areas. Braz J Infect Dis. 2015;19:146-55.

42. Martinez-Bello DA, Lopez-Quilez A, Torres PA. Relative risk estimation of dengue disease at small spatial scale. Int J Health Geogr. 2017;16:31.

43. Lin $\mathrm{CH}$, Wen $\mathrm{TH}$. Using geographically weighted regression (GWR) to explore spatial varying relationships of immature mosquitoes and human densities with the incidence of dengue. Int J Environ Res Public Health. 2011:8.2798-815.

44. Li Y, Kamara F, Zhou G, Puthiyakunnon S, Li C, Liu Y, et al. Urbanization increases aedes albopictus larval habitats and accelerates mosquito development and survivorship. PLoS Negl Trop Dis. 2014;8:e3301.

45. Zhu G, Xiao J, Zhang B, Liu T, Lin H, Li X, et al. The spatiotemporal transmission of dengue and its driving mechanism: a case study on the 2014 dengue outbreak in Guangdong, China. Sci Total Environ. 2018; 622-3:252-9.

46. Chen QQ, Meng YJ, Li Y, Qi XP. Frequency, duration and intensity of dengue fever epidemic risk in townships in Pearl River Delta and Yunnan in China, 2013. Biomed Environ Sci. 2015;28:388-95.

47. Sang $S$, Chen B, Wu H, Yang Z, Di B, Wang L, et al. Dengue is still an imported disease in China: a case study in Guangzhou. Infect Genet Evol. 2015:32:178-90.

48. Lin WX, Gao WF, Pu SX, Liu T, Lu ER. Correlations to estimate the monthly average daily diffuse radiation on horizontal surfaces in Yunnan Province, China. Energ Convers Manage. 1998;39:505-10.

49. Chen X, Qian G, Chen T, Li C, Yu K. Characteristics of climate warming and impact on climate zones change in Guangdong. J Trop Meteorol. 2007:13:61-4.

50. Zhang FC, Zhao H, Li LH, Jiang T, Hong WX, Wang J, et al. Severe dengue outbreak in Yunnan, China, 2013. Int J Infect Dis. 2014:27:4-6.

51. Wang BH, Li YP, Feng Y, Zhou HN, Liang YB, Dai JJ, et al. Phylogenetic analysis of dengue virus reveals the high relatedness between imported and local strains during the 2013 dengue outbreak in Yunnan, China: a retrospective analysis. BMC Infect Dis. 2015;15:142. 